$$
C_{i j}=\frac{N_{i j}\left\{\sum_{k=1}^{n} U_{x_{k}}^{(i)} N_{i k}\right\}^{(n-2) / 2}}{D^{(n-1) / 2}} .
$$

Now by a well known property of Jacobians, *

$$
\sum_{j=1}^{n} \frac{\partial C_{i j}}{\partial x_{j}}=0 .
$$

Hence, if in (19) the expressions on the right of (18) be substituted for $C_{i j}$, we will have the differential equation satisfied by $U^{(i)}$ alone. It is readily seen that the form of this equation is independent of the index ( $i)$ and hence the $n$ functions

$$
U^{(1)}, U^{(2)}, \cdots, U^{(n)}
$$

satisfy the same differential equation, which may be looked upon as a generalization of Laplace's equation to curved $n$-space.

WESLEYAN UNIVERSITY

\title{
THE NON-EXISTENCE OF A CERTAIN TYPE OF REGULAR POINT SET $\dagger$
}

\author{
BY R. L. WILDER
}

In a paper not yet published, $\ddagger$ I have shown that a regular $\S$ connected point set which consists of more than one point and remains connected upon the omission of any connected subset, is a simple closed (Jordan) curve. As a simple closed curve is a bounded point set, it is clear that there does not exist any unbounded regular connected point set which remains connected upon the omission of any connected subset.

* Muir, Theory of Determinants, vol. 2, p. 230.

$\dagger$ Presented to the Society, December 29, 1926.

¥ See, however, this Bulletin, vol. 32 (1926), p. 591, paper No. 35.

$\S$ That is, connected im kleinen. 
In the present paper I propose to consider the following question: Does there exist any unbounded regular connected point set which remains connected upon the omission of any bounded connected subset? If the additional restriction that it be closed is imposed upon the point set, J. R. Kline has shown* that the answer to this question is negative. In his proof Kline is able to make use of known properties of continuous curves. For the general case of non-closed sets these properties are not available, but by establishing certain properties of connected and regular point sets it is possible, as shown below, to give a negative answer to the above question.

Definition. If $M$ is a regular point set, a region of $M$ is defined as follows: $P$ being any point of $M$, and $C$ a circle with center at $P$, then the set of all points of $M$ which lie, with $P$, in a connected subset of $M$ which lies within $C$ is a region of $M$. It is clear that the set of all points of $M$ which lie in a certain neighborhood of $P$ are in a region of $M$.

Definition. If $A$ and $B$ are two distinct points of a regular set $M$, a simple chain of regions of $M$ from $A$ to $B$ is a finite sequence of regions of $M, R_{1}, R_{2}, \cdots, R_{n}$, such that (1) $R_{1}$ and $R_{2}$ contain $A$ and $B$, respectively, (2) $R_{i}(i \neq 1, n)$ has points in common with $R_{i-1}$ and $R_{i+1}$, but not with any other region of the sequence, (3) $R_{1}$ and $R_{n}$ have points in common with $R_{2}$ and $R_{n-1}$, respectively, but not with any other region of the sequence.

Theorem 1. If $A$ and $B$ are any two distinct points of a regular connected point set $M$, then there exists a simple chain of regions of $M$ from $A$ to $B$.

The proof of Theorem 1 is similar to the proof of Theorem 10 of R. L. Moore's On the foundations of plane analysis situs. $\dagger$

* Closed connected sets which remain connected upon the removal of certain connected subsets, Fundamenta Mathematicae, vol. 5 (1924), pp. 3-10.

$\dagger$ Transactions of this Society, vol. 17 (1916), pp. 131-164. 
THEOREM 2. If $M$ is a regular point set, then any region of $M$ is a regular point set.

THEOREM 3. If $M$ is a regular connected point set, bounded or unbounded, and $A$ and $B$ are any two distinct points of $M$, then both $A$ and $B$ lie in a bounded, regular, connected subset of $M$.

Theorem 3 is a consequence of Theorems 1 and 2 .

Theorem 4. Let $C_{1}$ and $C_{2}$ be two mutually exclusive point sets, and $M$ a regular connected point set which has at least one point in common with each of the sets $C_{1}$ and $C_{2}$, and such that the set of points common to $M$ and $C_{i}(i=1,2)$ is closed in $M$. Then there exists a point set $K$, subset of $M$, such that $K$ is connected and bounded and contains no point of either $C_{1}$ or $C_{2}$, but such that $C_{1}$ and $C_{2}$ each contain at least one point of $M$ which is a limit point of $K$.

Theorem 4 is a generalization of the result contained in my paper $A$ theorem on connected point sets which are connected im kleinen.* Its proof, after an application of Theorem 3 , is very similar to the proof of the result of the latter paper.

THEOREM 5. If $P$ is a point of a connected and regular point set $M$ such that $M-P$ is the sum of two mutually separated $\dagger$ sets $M_{1}$ and $M_{2}$, then $M_{1}+P$ and $M_{2}+P$ are connected and regular sets.

Proof. Let $K$ be any circle with center at $P$. Since $M$ is regular, there exists a circle $T$ concentric with $K$, such that all points of $M$ interior to $T$ lie with $P$ in a connected subset of $M$ which lies wholly interior to $K$. Denote by $k$ the set of all points of $M$ that lie with $P$ in a connected subset of $M$ which lies wholly interior to $K$, and by $t$ the set of all points of $M$ that lie interior to $T$. Clearly $t$ is a subset of $k$.

* This Bulletin, vol. 32 (1926), pp. 338-340.

$\dagger$ Two sets are said to be mutually separated if they are mutually exclusive and neither contains a limit point of the other. 
By a theorem due to Knaster and Kuratowski, ${ }^{*} M_{1}+P$ and $M_{2}+P$ are connected sets. Denote the set of points common to $k$ and $M_{i}(i=1,2)$ by $k_{i}$. Neither of these sets is vacuous, since both $M_{1}$ and $M_{2}$ have points in common with $t$, and hence with $k$. Clearly $k-P$ is the sum of the two mutually separated sets $k_{1}$ and $k_{2}$. That is, $P$ is a cutpoint of $k$.

It follows by the theorem of Knaster and Kuratowski referred to above that $k_{1}+P$ and $k_{2}+P$ are connected sets. If $x$ is any point of $M_{1}$ interior to $T$, then $x$ is a point of $k$ and a fortiori of $k_{1}$. Then there exists a connected subset of $M_{1}+P$, namely $k_{1}+P$, which contains both $x$ and $P$ and lies wholly within $K$. That is, the set of all points of $M_{1}+P$ which lie interior to $T$ lie, with $P$, in a connected subset of $M_{1}+P$ which lies wholly interior to $K$. Hence $M_{1}+P$ is regular at $P$. That it is regular at all other points is easily seen. Similarly, $M_{2}+P$ is regular.

THEOREM 6. If $M$ is a regular point set, $R$ a region of $M$, and $P$ a point of $R$, then if $k$ is a maximal connected subset of $R-P, k+P$ is a regular connected point set.

Proof. If $R-(k+P)$ is vacuous, $k+P$ is a regular connected set by Theorem 2. If $R-(k+P)$ is not vacuous, denote it by $q$. Then $R-P$ is the sum of the two mutually separated sets $k$ and $q$, and hence, by Theorem $5, k+P$ is connected and regular.

Definition. If $M$ is a point set, and $C_{1}$ and $C_{2}$ are mutually exclusive point sets, and $H$ is a connected subset of $M$ which has no point in common with either $C_{1}$ or $C_{2}$, but has limit points which are points of $M$ in both $C_{1}$ and $C_{2}$, then $H$, together with those points of $M$ in $C_{1}+C_{2}$ which are limit points of $H$, will be called a set $K\left(C_{1}, C_{2}\right) M . \dagger$ If the set

* B. Knaster and C. Kuratowski, Sur les ensembles connexes, Fundamenta Mathematicae, vol. 2 (1921), pp. 206-255, Theorem 6.

$\dagger$ I have made use of the sort of set defined here in other connections. See my paper $A$ property which characterizes continuous curves, Proceedings of the National Academy, vol. 11 (1925), pp. 725-728. Also see this Bulletin, vol. 32 (1926), p. 218, paper 4. 
$H$ is identical with that maximal connected subset of $M-M \times C_{1}-M \times C_{2}$ determined by $H$, then $H$ together with those points of $M$ in $C_{1}+C_{2}$ which are limit points of $H$, will be called a maximal set $K\left(C_{1}, C_{2}\right) M$ or, for the sake of brevity, a set $K^{\prime}\left(C_{1}, C_{2}\right) M$.

Definition. If $M$ is a regular point set, $P$ a point of $M$ and $C$ a circle enclosing $P$, then a branch of $M$ with respect to $P$ and $C$ is a set $K^{\prime}(P, C) M$.

THEOREM 7. There does not exist, in the plane, a regular, connected, unbounded point set which remains connected upon the omission of any bounded connected subset.

Proof. Suppose there does exist such a set. Denote it by $M$.

1. If $C$ is any circle, and $P$ a point of $M$ within $C$, say for convenience at the center of $C$, then I shall show that there exist infinitely many distinct branches of $M$ with respect to $P$ and $C$ such that any two of these are, except for $P$, mutually separated.

Let $S$ be the region of $M$ which is determined by $P$ and $C$. Since $M$ is regular, there exists a circle $T$ with center at $P$ such that all points of $M$ interior to $T$ lie in $S$. Denote the set of all points of $M$ interior to $T$ by $T_{1}$.

The set $S-P$ is not connected. For if it were, then $M-(S-P)$ would, by hypothesis, be connected, which is impossible since this set contains no point of $T_{1}$ except $P$. Then $S-P$ is the sum of two mutually separated sets $S_{1}$ and $S_{2}$. The sets $S_{1}+P$ and $S_{2}+P$ are connected, and by Theorems 2 and 5 are regular. If $x$ is a point of $S_{1}$, then by Theorem 4 the set $S_{1}+P$ contains a set $K(P, x)\left(S_{1}+P\right)$. Denote this set by $k_{1}$. The set $M-P$ being unbounded, connected (by hypothesis) and regular, contains a set $K(x, C)(M-P)$. Call this set $k_{2}$. Denote that portion of $k_{2}$ which is not on $C$ by $k_{2}^{\prime}$. The set $k_{2}^{\prime}$ is connected and, since it contains $x$, is a subset of $S_{1}$. Denote the set $k_{1}+k_{2}$ by $k$. Then $k$ is a set $K(P, C) M$. If to the set $k_{1}+k_{2}$ be added all those points of that maximal connected subset 
of $S-P$ determined by $x$, as well as the limit points of these points which are in $M$ and on $C$, the resulting set is a set $K^{\prime}(P, C) M$, which will be denoted by $K_{1}$. Denote the set of points $K_{1}-P-K_{1} \times C$ by $H_{1}$. Then $H_{1}$ is a connected subset of $S_{1}$.

In a similar way it can be shown that there exists a set $H_{2}$ which is a subset of $S_{2}$, and which, together with $P$ and its limit points on $C$ that belong to $M$, forms a set $K^{\prime}(P, C) M$ which will be denoted by $K_{2}$. The sets $H_{1}$ and $H_{2}$ are mutually separated.

(a) If $K_{1}-P$ and $K_{2}-P$ are not mutually separated, their sum forms a connected set $N$.

(b) If $K_{1}-P$ and $K_{2}-P$ are mutually separated, let the set of all points of $K_{i}(i=1,2)$ which lie between and on the circles $C$ and $T$ be denoted by $B_{i}$. The sets $B_{1}$ and $B_{2}$ are closed in $M-P$ and mutually exclusive. Hence, by Theorem 4 , there exists a set $K\left(B_{1}, B_{2}\right)(M-P)$ which is bounded. Denote this set by $N_{1}$. Then $\left(K_{1}-P\right)+\left(K_{2}-P\right)+N_{1}$ is a bounded connected subset of $M-P$ which will be denoted by $N$.

In either case (a) or (b), then, there exists a circle $G$ concentric with $C$, whose radius is greater than the radius of $C$, and which encloses a connected subset, $N$, of $M-P$, which contains $K_{1}-P$ and $K_{2}-P$. If $g$ is the region of $M$ determined by $P$ and $G$, then $g-P$ is the sum of two mutually separated sets $g_{1}$ and $g_{2}$, and $g_{1}+P$ and $g_{2}+P$ are connected and regular. As $N+P$ is connected and lies within $G$, it is clear that it is a subset of $g$. Hence $N$ is a subset of $g_{1}+g_{2}$ and being connected is a subset of one of the sets $g_{1}, g_{2}$, say $g_{1}$. As above, we can show that $M$ contains a set $k_{3}$ which is a branch of $M$ with respect to $P$ and $G$, and which, except for $P$ and its points on $G$, is a maximal connected subset of $g_{2}$. If $h_{3}$ denotes that portion of $k_{3}$ which is not on $G$, then the set $h_{3}+P$ is regular, by Theorem 6 , and hence contains a set $K^{\prime}(P, C) M$ which will be denoted by $K_{3}$. It is clear that the sets $K_{2}$ and $K_{3}$ are, except for $P$, mutually separated. Denote the set $K_{3}-P-K_{3} \times C$ by $H_{3}$. 
By means of Theorem 4, it can be shown that there exists a circle $G_{1}$ of radius greater than the radius of $G$, such that the sets $K_{i}-P(i=1,2,3)$ all lie in a connected subset, $F$, of $M$, which lies within $G_{1}$. If $q$ is the region of $M$ determined by $P$ and $G_{1}$, then $q-P$ is the sum of two mutually separated sets, $q_{1}$ and $q_{2}$, one of which, say $q_{1}$, contains $F$. Then a subset $K_{4}$ of $q_{2}+P$ may be found which is a set $K^{\prime}(P, C) M$.

Continuing as indicated above, the existence of an infinite sequence of sets $K_{2}, K_{3}, K_{4}, \cdots$, such that for every positive integer $n>1, K_{n}$ is a set $K^{\prime}(P, C) M$, and such that any two of these sets are, except for $P$, mutually separated, is established.

2. Consider in particular the sets $K_{2}, K_{3}, K_{4}$, and $K_{5}$. For each $i,(i=2,3,4,5)$, let $A_{i}$ be a point of $K_{i}$ on $C$. Two of these points must separate the other two on $C$; say $A_{2}$ and $A_{3}$ separate $A_{4}$ and $A_{5}$ on $C$. As $M-\left(K_{4}+K_{5}\right)$ is connected, by hypothesis, and regular since $K_{4}+K_{5}$ is closed in $M$, it follows that there exists, by Theorem 4 , a bounded set $K\left(\dot{A}_{2}, A_{3}\right)\left(M-K_{4}-K_{5}\right)$ which, together with the set of points $K_{2}+K_{3}-P$, is a bounded connected subset $V$ of $M-P$. There exists a circle $E$ concentric with $C$, which encloses $V$ and contains no limit points of it.

Just as the existence of branches of $M$ with respect to $P$ and $C$ were established, it can be shown that there exists an infinite set $K_{i}\left(A_{j}\right),(i=1,2,3, \cdots, j=4,5)$, of branches of $M$ with respect to $A_{j}$ and $E$. From the definition of a branch of $M$, it is clear that the connected set $V+K_{4}+K_{5}$ must lie wholly in one branch of $M$ with respect to $A_{4}$ and $E$, say in $K_{1}\left(A_{4}\right)$, and in one branch of $M$ with respect to $A_{5}$ and $E$, say $K_{1}\left(A_{5}\right)$.

As the set $V$ contains no limit points of the set points $K_{4}+K_{5}+K_{2}\left(A_{4}\right)+K_{2}\left(A_{5}\right)$, every point of it is the center of a circle which neither encloses any point of the latter set nor of $E$, nor has any point in common with either. The sum of the interiors of all such circles is a connected domain and this domain contains an arc $t_{1}$ from $A_{2}$ to $A_{3}$. Clearly $t_{1}$ and $K_{4}+K_{5}+K_{2}\left(A_{4}\right)+K_{2}\left(A_{5}\right)$ are mutually separated. 
The points sets $K_{2}+K_{3}$ and $K_{2}\left(A_{4}\right)+K_{2}\left(A_{5}\right)$ are mutually separated. Denote by $U$ the set of points consisting of $K_{2}\left(A_{4}\right)+K_{2}\left(A_{5}\right)$ together with its limit points. Then $K_{2}+K_{3}$ and $U$ are mutually exclusive. Let $C\left(A_{2}\right)$ and $C\left(A_{3}\right)$ be circles with centers at $A_{2}$ and $A_{3}$, respectively, and enclosing no point of $U$. If $a_{i}(i=2,3)$ is a point of $H_{i}$ lying within $C\left(A_{i}\right)$, there exists an arc $b_{i}$ joining $A_{i}$ and $a_{i}$ which lies entirely within $C\left(A_{i}\right)$, and except for $A_{i}$ lies wholly within $C$. As $H_{2}+H_{3}+P$ is a connected subset of $C$ containing $a_{2}$ and $a_{3}$ but no point of $U$, there exists, by Theorem $\mathrm{H}$ of my paper On a certain type of connected set which cuts the plane,* an arc $b_{1}$ which joins $a_{2}$ and $a_{3}$, contains no point of $U$, and lies wholly within $C$. Clearly the continuous curve consisting of the $\operatorname{arcs} b_{1}, b_{2}$, and $b_{3}$ contains an arc $t_{2}$ which joins $A_{2}$ and $A_{3}$, lies except for these points entirely within $C$, and contains no point of $U$. Similarly, since $K_{4}+K_{5}$ and $t_{1}$ are mutually separated, there exists an arc $t_{3}$ joining $A_{4}$ and $A_{5}$, lying except for these points entirely within $C$, and having no point in common with $t_{1}$.

By the corollary to Theorem D of the paper referred to in the preceding paragraph, $\dagger$ there exists a simple closed curve $J$ which is a subset of $t_{1}+t_{2}$ and separates the plane between $A_{4}$ and $A_{5}$. However, $J$ has no points in common with either $K_{2}\left(A_{4}\right), K_{2}\left(A_{5}\right)$ or $E$, and yet the sum of these three sets is a connected set containing $A_{4}$ and $A_{5}$. Thus a contradiction is established and the theorem is proved.

The University of Michigan

* To appear in the Proceedings of the International Mathematical Congress at Toronto. Theorem $\mathrm{H}$ of this paper is the following: Let $G$ be a bounded domain, $K$ any closed set of points and $N$ a connected subset of $G$ which contains no points of $K$. Then every pair of distinct points of $K$ are the end-points of an arc which lies in $G$ and contains no points of $K$.

$\dagger$ The corollary referred to here is the following: If $A$ and $B$ separate $C$ and $D$ on a simple closed curve $K, A B$ and $C D$ are arcs joining $A, B$ and $C, D$, respectively, and lying, except for their end-points, interior to $K$, and $t$ is an arc from $A$ to $B$ that contains no points of $C D$, then there exists a simple closed curve $J$ which is a subset of $A B+t$, such that $C$ is interior to $J$ and $D$ exterior to $J$, or vice versa. 